AIAA 2001-3813

\title{
MODELING OF MULTI-TUBE PULSE DETONATION ENGINE OPERATION ${ }^{1}$
}

\author{
Houshang B. Ebrahimi ${ }^{*}$, Rajendran Mohanraj ${ }^{*}$ and Charles L. Merkle ${ }^{\dagger}$ \\ University of Tennessee Space Institute \\ 411 B. H. Goethert Parkway \\ Tullahoma, TN 37388-9700, USA
}

\begin{abstract}
The present paper explores some preliminary issues concerning the operational characteristics of multipletube pulsed detonation engines (PDE's). The study is based on a two-dimensional analysis of the first-pulse operation of two detonation tubes exhausting through a common nozzle. Computations are first performed to assess isolated tube behavior followed by results for multi-tube flow phenomena. The computations are based on an eight-species, finite-rate transient flowfield model. The results serve as an important precursor to understanding appropriate propellant fill procedures and shock wave propagation in multi-tube, multi-dimensional simulations. Differences in behavior between single and multi-tube PDE models are discussed. The influence of multi-tube geometry and the preferred times for injecting the fresh propellant mixture during multi-tube PDE operation are studied.
\end{abstract}

\section{Introduction}

A pulse detonation engine (PDE) is an unsteady propulsive device that utilizes the pressure rise from a detonation wave to reduce pumping requirements without sacrificing overall engine pressure ratio capability. In its simplest form, the PDE device is composed of a long tube (the detonation tube) that is closed at one end and open at the other. The propellants are fed into the tube through valves placed at or near the closed end, or distributed along the length of the tube. The cycle starts by filling the tube with fuel and oxidizer. A critical issue in the fill process is to achieve a sufficiently uniform mixture that a detonation can be sustained throughout the tube. After the fill process is completed, a spark initiator or other device placed at some appropriate location in the tube initiates a detonation. Practical implications favor initiation from the closed end, although alternative locations provide similar thermodynamic performance. In typical applications, a non-trivial distance is required to achieve transition to detonation. Once established, the detonation travels at speeds of a few kilometers per second into the combustible mixture, converting it to products in a nearly constant volume manner. The hot gases produced by the detonation are then allowed to exhaust through the open end of the tube during a blowdown phase to provide thrust. Following blow-down, the valves are again opened to fill the tube and the process repeats.

Reviews of previous work on PDE's can be obtained from Eidelman et al. ${ }^{1}$, and Bussing and Pappas. ${ }^{2}$. A recent summary of the status of current research on PDE's is given by Kailasanath et.al ${ }^{3}$. Cambier and Tegner ${ }^{4}$ discuss issues related to optimization of PDE performance. The review by Kailasanath ${ }^{5}$ deals with

"Adjunct Professor, Member AIAA

- Post Doctoral Research Associate, Member AlAA

+ Professor, H.H. Arnold Chair in Computational Mechanics, Member AIAA

'Copyright 12001 by the American Institute of Aeronautics and

Astronautics, Inc. All rights reserved 
application of detonation in propulsion and includes the research developments in PDE's. Most analytical studies of PDE's have dealt with one-dimensional models ${ }^{1.4 .7-10.13}$, although these have been augmented by both two-dimensional models, ${ }^{42,10}$ and zerodimensional models ${ }^{11,10}$. Most previous PDE studies have focussed on gaseous applications although some recent works ${ }^{6}$ involve liquid fuels such as JP- 10 .

The impulse produced by a single pulse of a PDE is typically quite low. The time-averaged thrust can be increased by using a larger detonation chamber volume, by operating at higher frequencies or by operating at increased pressure levels. An alternate way for increasing thrust is to combine multiple detonation tubes into a single engine. The individual tubes within the engine can be fed from a common inlet and discharged into a common nozzle. The use of a common inlet and nozzle may provide improved weight characteristics while also reducing the degree of unsteadiness in these components and the overall level of engine vibration.

In air-breathing applications, using the air supply from a common inlet to fill multiple detonation tubes reduces the drag associated with the inlet during time intervals in the cycle when none of the detonation tubes are being filled ${ }^{13}$ and mitigates against possible unstart. Feeding the exhausts from multiple detonation tubes into a common nozzle reduces the degree of unsteadiness in the thrust and appears to improve nozzle performance. A divergent nozzle section added to a single-tube PDE will act as a diffuser rather than a flow accelerator during large portions of the cycle when the total pressure in the unsteady exhaust stream becomes too low. Multiple tubes discharging into a common nozzle provide higher average total pressures that help to ensure that the nozzle accelerates the flow over a larger fraction of the PDE cycle. In addition, the throat of the common nozzle can be chosen to provide an effective back-pressure at the exit of the detonation tubes that increases their average operating pressure and enables them to generate more thrust. Finally, the use of multiple detonation tubes may also enable thrust vectoring and a wider throttling range.

In our previous work $^{7-10}$ we have used zero dimensional, one-dimensional and two-dimensional unsteady models of a single tube PDE manner to elucidate the important physics in a PDE and to document expected performance ranges. In addition, multi-tube PDEs have been investigated by means of a simplified one-dimensional model ${ }^{14}$. The results show that the fill process in a multi-tube PDE can be markedly different from that for a single tube. The multi-tube PDE can demonstrate a saturation effect in which increasing the fill time does not affect the fill fraction in the tubes. The conditions at which this occurs depend on the parameters such as the common nozzle throat area, the period, and the back pressure.

In the present paper, we use two-dimensional analyses to study multiple detonation tubes firing into a common nozzle, but for reasons of computational efficiency, focus on first-pulse results rather than periodic operation. Before presenting the multi-tube results, we first present some new single tube results to document the dominant exhaust characteristics of an isolated detonation tube. We then extend these single-tube analyses to a multi-tube PDE environment. The present two-dimensional, multi-tube analysis serves as a precursor for understanding the flow-field behavior in a multi-tube/common nozzle system and for developing a strategy for implementing repetitively pulsed multi-tube computations. An $\mathrm{H}_{2} / \mathrm{O}_{2}$ chemical kinetics system comprised of eight chemical species $\left(\mathrm{O}, \mathrm{O}_{2}, \mathrm{OH}, \mathrm{H}\right.$, $\mathrm{HO}_{2}, \mathrm{H}_{2} \mathrm{O}, \mathrm{H}_{2} \mathrm{O}_{2}$, and $\mathrm{H} 2$ ) and 16 reactions was used in all of the calculations.

\section{Model Formulation}

The flow characteristics in a pulse detonation engine have been modeled previously using a variety of methods $s^{2.7}$ including zero-dimensional ${ }^{10.11}$, onedimensional ${ }^{7-10}$ and two-dimensional unsteady analyses $^{10.12}$. All three of these levels are useful, but provide different types of information. Zerodimensional analyses provide fast, global parametric trends for the unsteady operation of a PDE. Onedimensional models provide a first indication of the dominant wave processes and the manner in which they couple with the overall engine/vehicle system at a cost that is intermediate between zero- and two-dimensional models. Two-dimensional models have the capability for identifying the dominant multi-dimensional effects and their level of importance. Multi-dimensional modeling, however, requires a substantial investment of computational resources so that the allowable number of computational is typically quite limited. Some specific areas of PDE operation are, however, inherently dominated by multi-dimensional phenomena, and for assessing such phenomena, the modeling must invoke multi-dimensional computations.

\section{Single-Tube Results}

To provide background for understanding the flowfield in a multi-tube PDE we first look briefly at representative results from two dimensional computations of an isolated detonation tube exhausting into a large unconfined region. The isolated tube computations involve single pulse detonations starting from an ambient pressure of $1.0 \mathrm{~atm}$. The pressure in 
the external environment is likewise set at $1.0 \mathrm{~atm}$. As with our later multi-tube results, these single-tube computations are based on a detailed hydrogen-oxygen kinetics mechanism ${ }^{7}$ and are for a mixture at stoichiometric conditions. A parametric set of results has been obtained for three different detonation tube lengths $-0.06 m, 0.12 m$ and $0.18 m$.
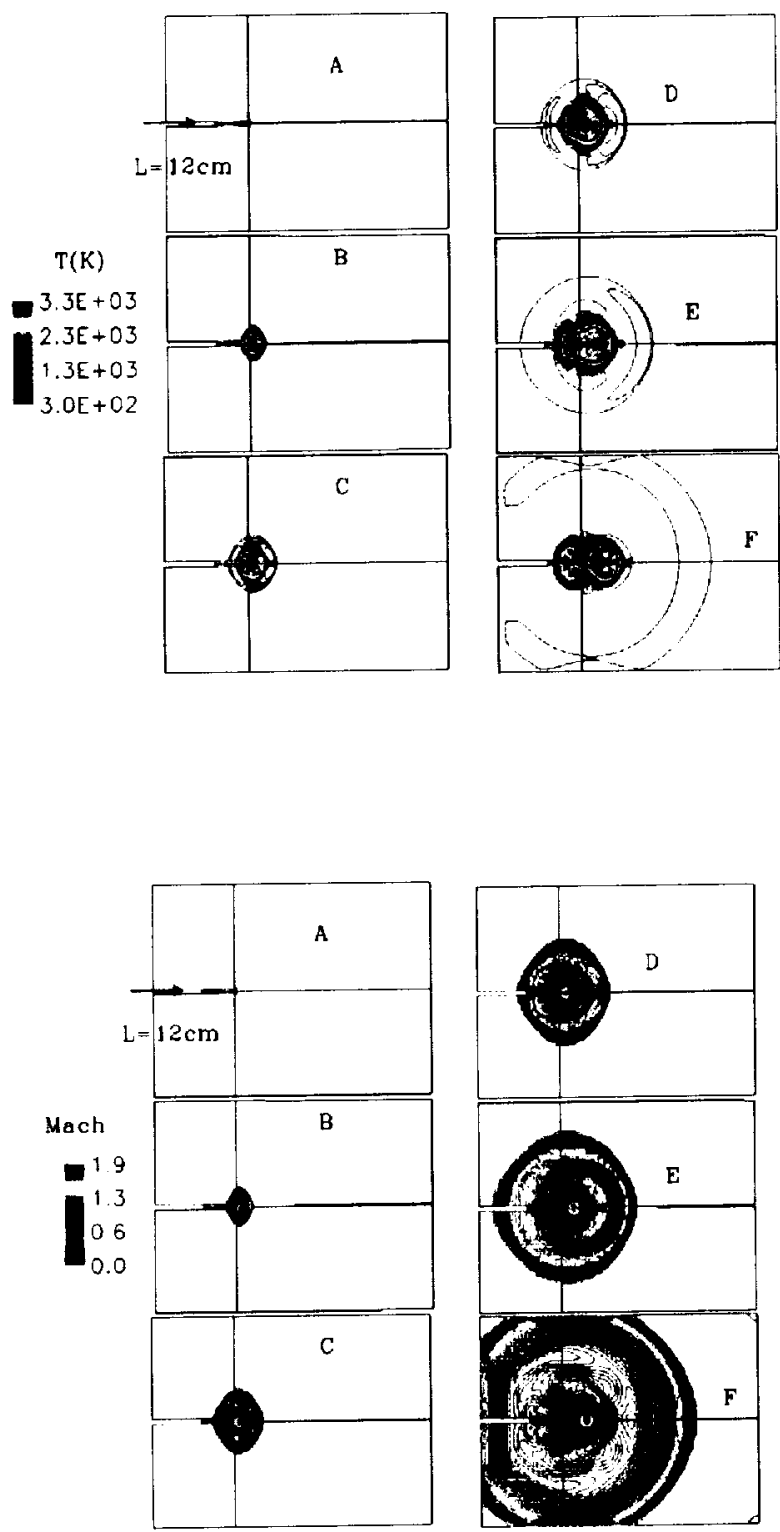

Fig. 1. Temporal devlopment of external flowfield behind $12 \mathrm{~cm}$ tube. Upper plots: Temperature. Lower Plots: Mach number.

The flowfield generated by an isolated detonation tube can be separated into two regions, one inside the tube and the other outside. The internal flowfield is initially characterized by the dynamics of the detonation as it moves from the initiation zone at the closed (left) end to the open (right) end of the tube. At later times the internal flow is characterized by reverberation processes as the pressure seeks to equilibrate with conditions outside the tube.

The external flowfield is the result of the expansion of the high-pressure detonation products through the open end of the tube and into the external environment. In general the external flow is composed of the shock wave created by the detonation and the ensuing compression and expansion processes. One of the purposes of the multiple tube arrangement is to control the dynamics of this 'external' field in such a way that the overall performance (impulse and thrust to weight) of the engine is improved.

A time sequence of the external flowfield behind the 12 $\mathrm{cm}$ tube is given in Fig. 1. The plots in the upper portion of the figure show the temperature contours. Those in the lower portion show the Mach number contours. Representative long-term results for all three tube lengths are shown in Fig. 2, this time showing the pressure and the Mach number. Other more detailed studies of single- tube PDE operation based on the present model have been reported previously ${ }^{7.10}$.

The results in Figs. 1 and 2 clearly show the approximately cylindrical shock wave that is generated as the detonation emerges from the open end of the tube. In general, this shock wave remains symmetric about the tube axis as it propagates away from the exit plane of the tube. The strength of the shock decreases steadily as it moves away from the tube and its radius increases. The pressure plots in Fig. 2 also show that the aft-portion of the external shock is considerably stronger than the forward portion. (The shock reflection from the top and bottom of the $18 \mathrm{~cm}$ tube case occur because the outer boundary of the finite domain was treated as a solid wall.) Note that the expanding shock wave creates a large sub-atmospheric pressure region in the vicinity the nozzle exit plane. This low pressure region has a major impact on the establishment of reverse flow at the exit and the ensuing reverberation process inside the tube.

Clearly the external flow calculations for this case remain meaningful only so long as there are no external surfaces available that the flow must pass over. In the case where multiple tubes are placed in proximity with each other, the expansion from one tube will clearly impact conditions in adjacent tubes. If in addition to placing multiple tubes adjacent to each other, their exhaust is coupled into a common convergingdiverging nozzle, this external flow will likewise reflect 
from the nozzle surfaces producing additional interactions. Finally, the presence of the nozzle will cause each detonation tube to see an unsteady, fluctuating pressure and, depending on the size of the nozzle throat will raise the average effective back pressure. An assessment of such factors are the primary focus of the present paper.
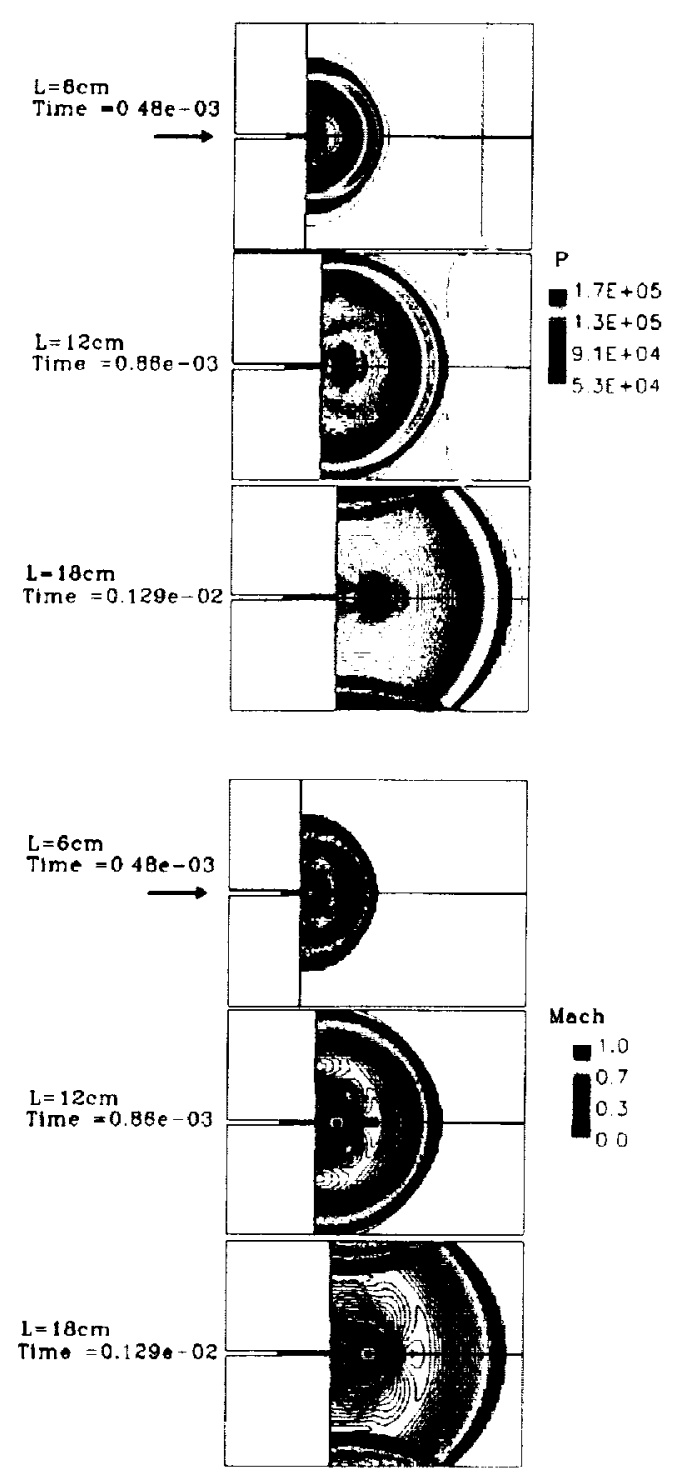

Fig. 2. Pressure and Mach number contours created by exhaust from isolated detonation tubes of three different lengths: $6 \mathrm{~cm}, 12 \mathrm{~cm}$, and $18 \mathrm{~cm}$.

\section{Multi-Tube Model and Geometry}

The unsteady, two-dimensional model has also been used to understand the operation of a multi-tube PDE with a common nozzle and to account for the effects of multiple detonation tubes exhausting through a common nozzle. A schematic of the multi-tube configuration is shown in Figure 3.

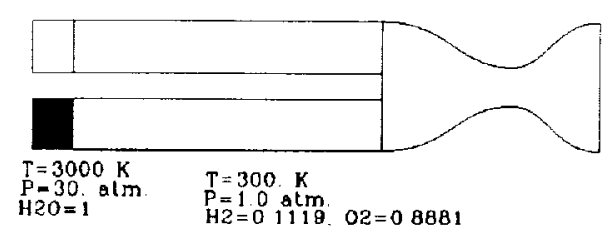

Fig. 3. Schematic of Multi-tube Configuration

Preliminary multi-tube PDE computations have been performed for a parametric series of five cases. To minimize computational requirements, all solutions reported herein are for a pulsed detonation engine consisting of two tubes whose open ends are connected to a common converging-diverging nozzle. Engines composed of as many as five or six tubes exhausting into a common nozzle are of interest in practical applications, but the present two-tube model provides an initial indication of the types of interactions that will occur when multiple tubes are combined in this fashion. For simplicity in this initial study of the effects of a common nozzle, we have chosen to use twodimensional geometries to avoid the strongly threedimensional effects that occur when multiple tubes are joined to an axisymmetric nozzle. The qualitative features of the two-dimensional configuration are expected to be similar to those of the axisymmetric configuration although quantitative features will be different.

Approximate one-dimensional analyses of multi-tube operation $^{14}$ indicate that it takes several cycles before periodic operation is attained in a multi-tube configuration. Further, the ultimate pressure level in the individual detonation tubes depends upon the number of tubes, their volume, mass per pulse and individual pulse repetition rate, and the nozzle throat area. The number of cycles needed to attain periodicity in a multi-tube configuration is typically larger than that needed to attain periodicity for single tube operation. In the present analyses, we consider only first-pulse detonation, not the periodic case.

When the detonation reaches the exit of a tube in a multi-tube PDE configuration, it causes a sudden pressure rise at the nozzle exit plane that results in reverse flow at the exit of the other detonation tubes where the pressure is still low. A multi-tube PDE involving a common nozzle causes a reverse flow to occur in the detonation tubes during some portions of the cycle, and the minimum pressure level in the cycle 
appears to be higher than the corresponding value for a single tube PDE.

The geometry of the configurations considered in the present computations is as follows (refer to Fig. 3. The length and diameter of each tube is $0.1 \mathrm{~m}$ and $0.01 \mathrm{~m}$, respectively. The tubes are spaced one-half tube width $(0.05 \mathrm{~m})$ apart, and are connected to a convergingdiverging nozzle by means of a smooth contour here chosen as a second-derivative-continuous cubic section. The throat area in three of the computations was taken equal to one-half the cross-stream area of an individual tube (a throat height of $0.005 \mathrm{~m}$ ), while for the other two it is taken as three-fourths of their area $(0.0075 \mathrm{~m})$. In all cases the throat was placed at an axial distance of two tube heights downstream of the exit plane of the detonation tubes. As in the above results, a stoichiometric hydrogen-oxygen mixture at an initial pressure of $1.0 \mathrm{~atm}$ was used for all multiple tube results.

The five cases presented are comprised of one in which detonations are initiated in both tubes simultaneously; two cases (one for each throat area) in which a detonation is initiated in only one tube; and two cases in which detonations are initiated in both tubes in a sequential, rather than simultaneous, manner. Representative results from these computations are discussed below.

\section{Multiple-Tube Results}

Simultaneous Initiation in Both Tubes: As a first case we consider the flowfield produced by simultaneously initiating detonations inside both tubes. Simultaneous detonations are not of practical interest, but give a first assessment of the manner in which the unsteady detonations interact with the nozzle while also providing confidence in our calculations. In addition, these computations provide a contrast with our isolated tube calculations. Because of our interest in the nonsymmetric flowfield created by sequential detonations in the two tubes, the full two-tube domain is computed rather than imposing a symmetry plane and computing only half the domain.

For this simultaneous initiation case, both tubes are initially filled with a stoichiometric mixture of hydrogen and oxygen at time zero while the converging and diverging portions of the nozzle contain pure oxygen. The oxygen in the nozzle does not lead to additional reaction when stoichiometric mixtures are used in the detonation tubes, and so serves as an effective inert. The initial pressure is one atmosphere throughout the entire pulsed detonation engine. Simultaneous detonations are initiated in both tubes by raising the pressure and temperature inside the 'initiation' region described above and setting the products there to pure water (see Fig. 3). Corresponding color contour plots of both the pressure and Mach number for this simultaneous initiation case are presented in Fig. 4 for six different time frames corresponding to conditions in the first 200 $\mu s$ following initiation.
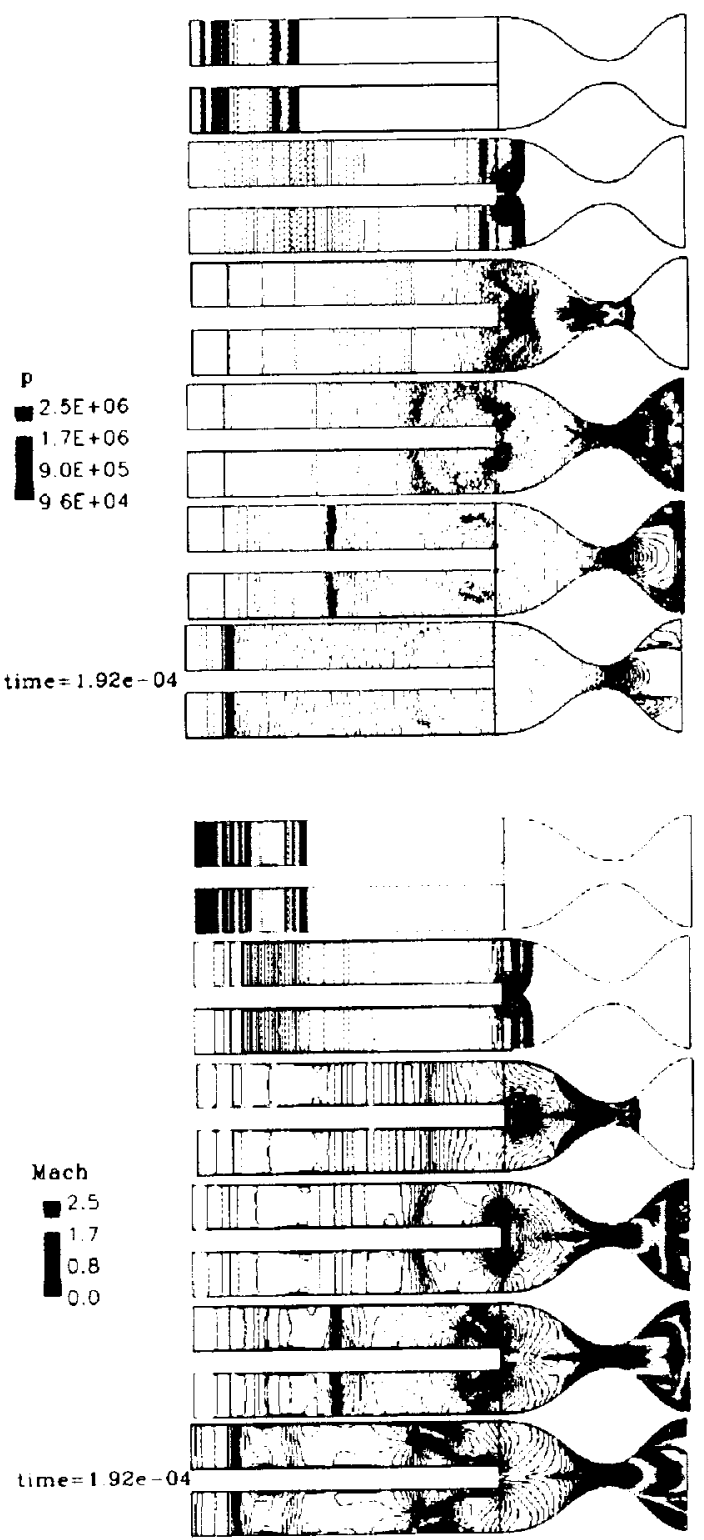

Fig. 4. Time history of pressure and Mach number contours for dual-tube pulsed detonation engine. Both tubes fired simultaneously. Small throat area.

In Frame $\mathrm{A}$ of Fig. 4, the detonation has traveled approximately one-third the distance down the tubes. The rarefaction wave has yet to traverse the entire initiation zone, so the pressure at the head end remains 
equal to the $30 \mathrm{~atm}$ initiation pressure. The location of the detonation is clearly seen in both tubes.

In Frame $B$ the detonations have just exited from the tubes and have merged into a nearly planar shock wave. Since the medium inside the nozzle is pure oxygen, no reaction occurs and the detonation rapidly transitions to a shock wave. The results in this frame remain nearly one-dimensional in character. The early-time behavior of the simultaneous detonation problem is nearly identical to that of the isolated tube shown above, but begins to deviate rapidly as the shock waves reflect from the internal surfaces of the nozzle.

As the shock continues toward the nozzle throat, the converging geometry enhances the strength of the shock such that a high-pressure region is seen near the throat, with a considerable amount of two-dimensional structure as shown in Frame C. At this instant, the shock is just beyond the throat and is propagating toward the exit plane.

Conditions after the shock reaches the exit plane are given in Frames D, E, and F. Comparison of these Frames with Frame $\mathrm{C}$ indicates a continual reduction of pressure at the nozzle exit plane following the transmission of this initial shock. A large fraction of the divergent passage is, however, filled with supersonic flow throughout this period. Also note that the Mach number contours show significant twodimensional structure near the downstream end of the tubes as reflections from the nozzle are transmitted back into the tubes. Corresponding pressure-time plots on the upstream end indicate that the pressure on this end increases more rapidly in the presence of a C-D nozzle than when an isolated tube is fired.

Results with Small Throat Area: In the second case, a detonation was initiated in only the lower tube and the ensuing reverberation of the pulse in both tubes and in the C-D nozzle was observed. This case represents the smaller throat diameter where the throat area is half of the detonation tube area. The results for Case 2 are shown in Fig. 5, and again, pressure and Mach number contours are given for each of six different times. The total time simulated in this computation is $275 \mu \mathrm{s}$ which is slightly longer than that in the first case.

Frame A of Fig. 5 corresponds to the time just after the detonation has emerged from the tube. Prior to this time conditions inside the lower tube are identical to those in Case 1. Note that the time for Frame A in this figure is approximately the same as that corresponding to Frame B in Fig. 4. The results clearly exhibit strong two dimensionality as the shock wave diffracts around the land area between the tubes causing the flow to begin to enter the upper tube. Simultaneously the shock wave is propagating into the converging section of the nozzle where additional two-dimensional effects are observed.

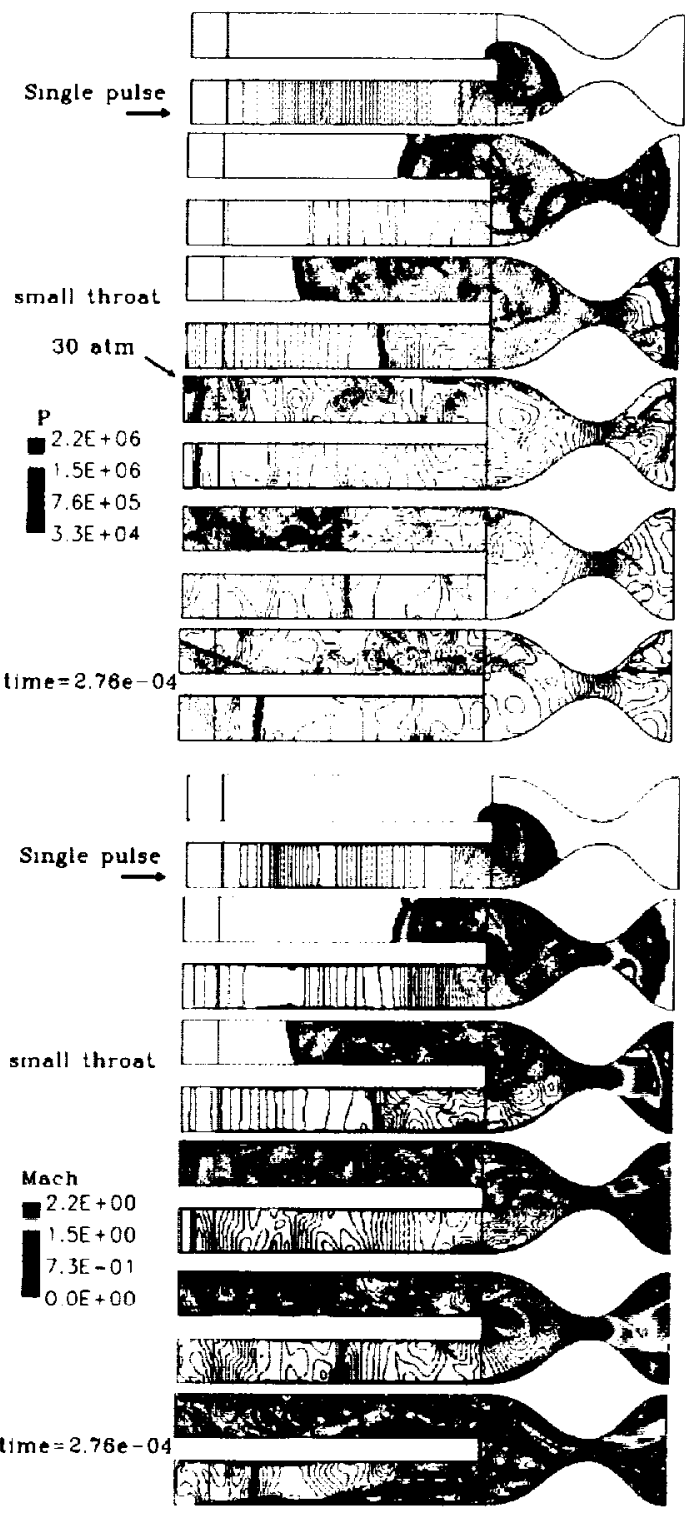

Fig. 5. Time history of pressure and Mach number contours for single detonation pulse in the bottom tube. Small throat area.

At a slighter later time (Frame B) the shock wave created by the detonation in the lower tube has propagated nearly half way inside the upper tube and through the nozzle throat and into the divergent section. Both the pressure and Mach number contours indicate substantial asymmetries in the flow in the divergent section that are much stronger than those observed in isolated detonation tubes, or in the simultaneous initiation case shown in Fig. 4. 
In Frame $\mathrm{C}$, the shock in the upper tube has traversed about $75 \%$ of the tube length and is nearing the upstream end. The flow in the divergent section remains quite asymmetric but still shows a considerable amount of supersonic flow. The shock reflection from the closed end of the upper tube takes place between Frames $C$ and $D$. In Frame $E$, the pressure on the upstream wall of the upper tube is nearly as high as that produced by the detonation in Frame A. Additional details of this are given later. The strength of the shock reflection off the end-wall of the non-detonated tube is somewhat overstated for this relatively short tube. As the tube length is increased (with no change in the nozzle section) expansion waves would overtake and weaken the shock before it reflects from the head end so that this reflected pressure would be reduced. Additional calculations are needed to ascertain the manner in which tube length will affect the results.

In the final frame (Frame $F$ ) the pressure on the upstream end of the upper tube has decayed considerably, but remains substantially above ambient conditions. These results suggest that the determination of an optimum time for starting the fill process and initiating a detonation in the upper tube may require considerable effort. The shock reflection in the upper tube produces a considerable pressure spike on this wall. In addition, the peak pressure in the tube that has not been fired occurs at a later time than in the tube that has been fired. The high pressure in the upper tube makes it difficult to fill that tube with a fresh propellant mixture. In fact, it is possible that the lower tube may return to conditions compatible with filling and refiring before the upper tube does. Certainly, if two (or more) tubes are to be used, it is imperative that all tubes be fired in sequence. The interplay between tubes appears to make sequencing difficult (but also makes it very important) and requires further study beyond the present manuscript. An initial estimate of successive firing of the two tubes is given next in Case 3.

The computations in Case 3 are identical to those in Case 2 except that a detonation was initiated in the upper tube just after the detonation in the lower tube emerged from the tube exit. The pressure and Mach number contours for this case are given in Fig. 6. The results in Frame A correspond to conditions just after initiation in the lower tube. Frame $B$ shows conditions just after the detonation in the upper tube has been initiated. The detonation in the lower tube has traveled just beyond that corresponding to Frame $A$ in Case 2 (Fig. 5). The primary distinction between the results in this present calculation and those in Frame A of Fig. 4 is that here the upper tube has been filled with a combustible mixture of hydrogen and oxygen just prior to this time frame. Detailed study of additional frames (not shown here for conciseness) indicates that in the interval between Frames $B$ and $C$ the shock wave from the lower tube induces a second detonation-like pulse from the open end of the upper tube. The remnants of this forward-propagating detonation are visible in the Mach number contours of Frame $C$. The net result of this second detonation appears to lead to a reduction in the pressure on the head end of the upper tube. Overall, the effects of sequential detonations in the two tubes appears to reduce the pressure signature on the upstream end of the upper tube as compared to the detonation of an isolated tube. In addition, we see that two dimensional effects are again more significant in this two-detonation problem than in the singledetonation case of Fig. 5.
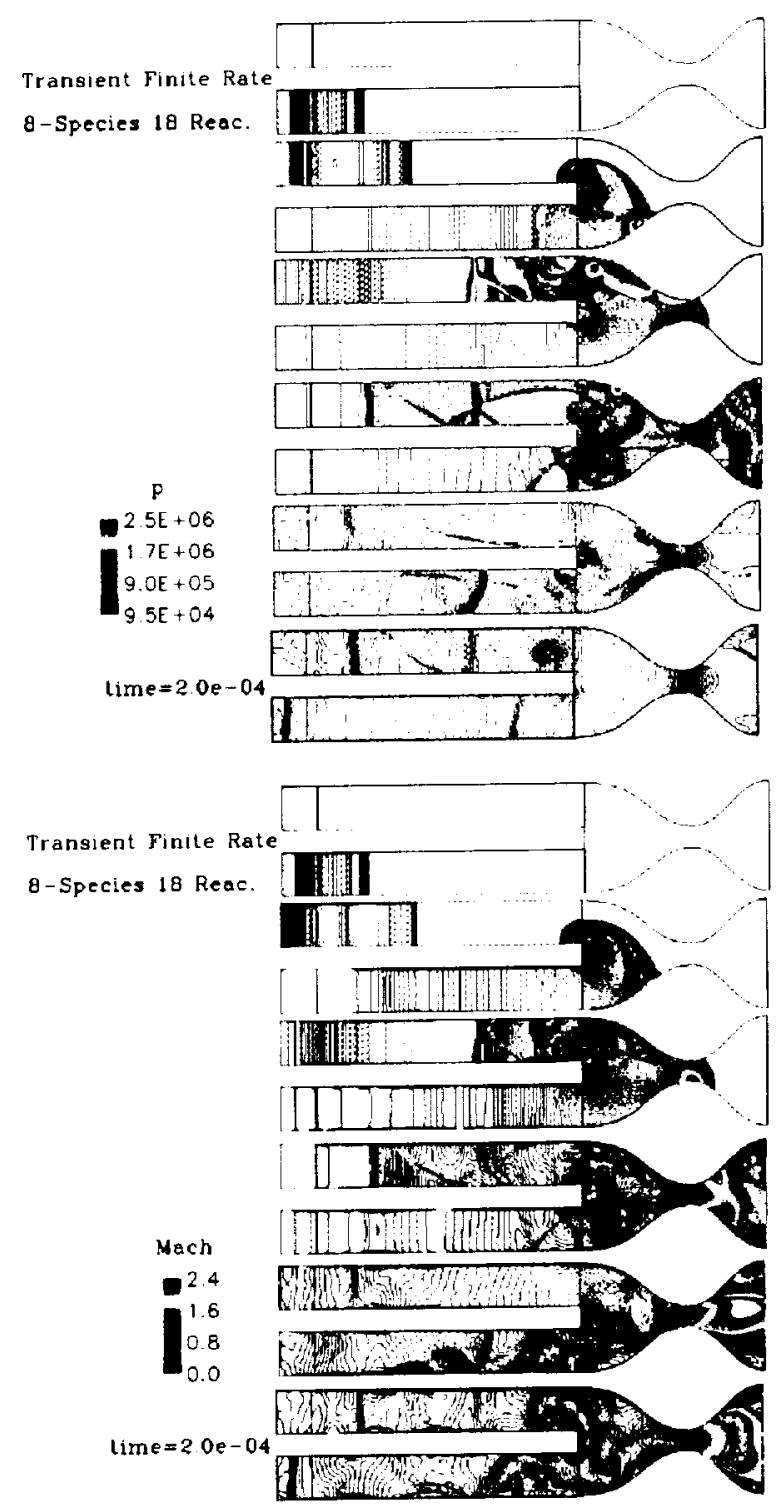

Fig. 6. Time history of pressure and Mach number contours for dual-tube pulsed detonation engine. Detonation in upper tube initiated when detonation in lower tube enters common nozzle. Small throat area. 

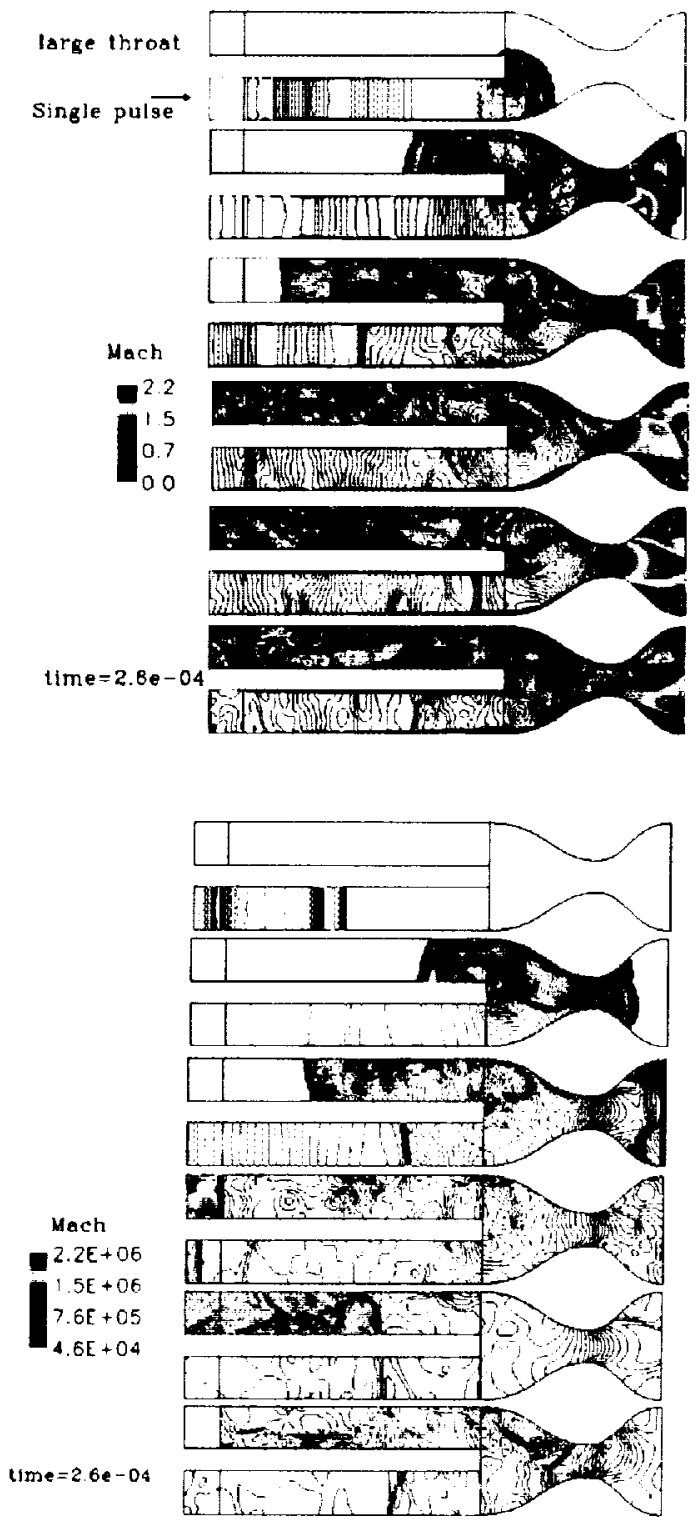

Fig. 7. Time history of pressure and Mach number contours for single pulse detonation for larger throat.

Results for Large-Throat Area: The results of the fourth case (Fig. 7) are similar in nature to the singlepulse results of Case 2 except that here the nozzle area has been increased by $50 \%$ so that it now corresponds to $75 \%$ of the cross-sectional area of a single tube. This allows the effects of the initial pulse to pass through the throat more readily and reduces the interactions between the two tubes. In the presence of this larger throat, the pressure decay across both tubes due to the detonation in the lower tube occurs faster than for the smaller throat case.

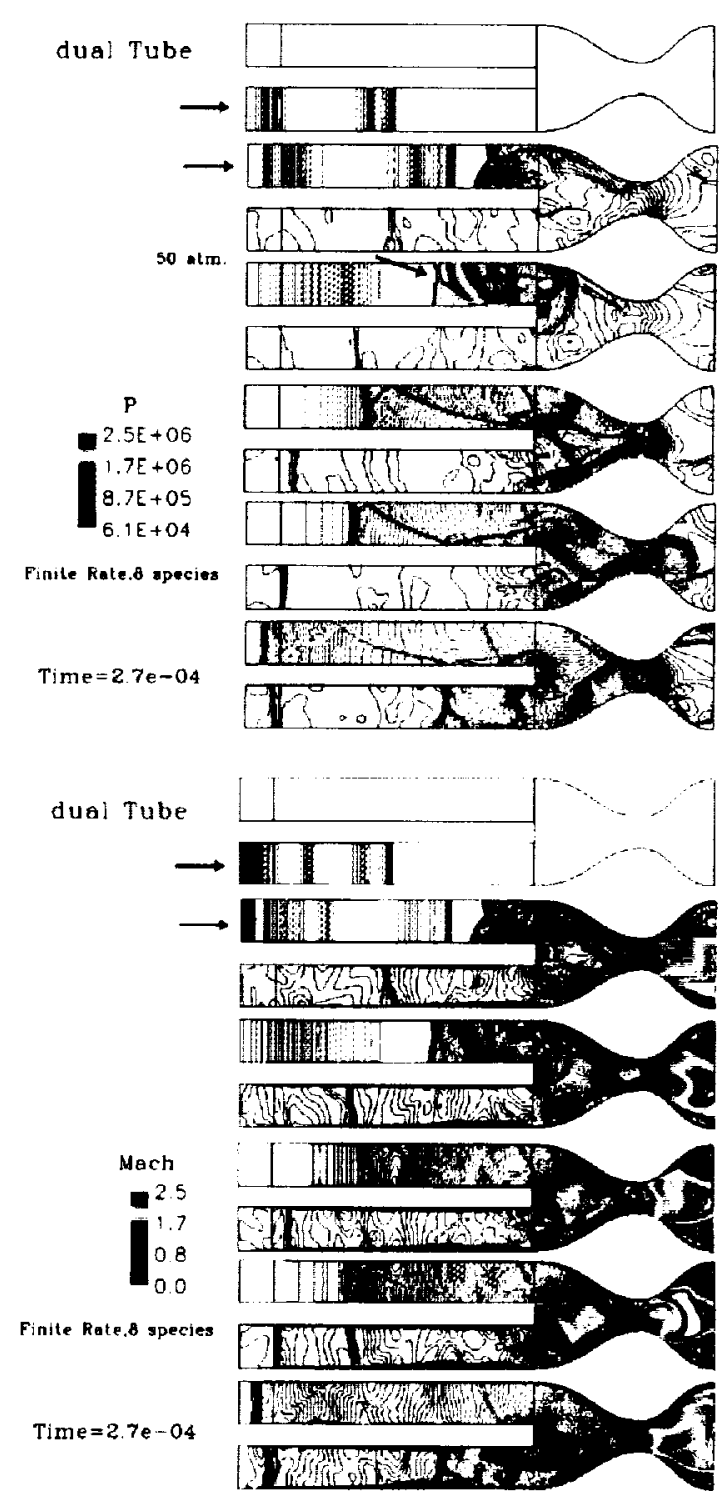

Fig. 8. Time history of pressure and Mach number contours for larger throat configuration. Sequential detonations in both lower and upper tubes.

Comparison between the results on Fig. 7 and those on Fig. 5 shows the impact of increasing the throat area. Note that in general the larger area results in smaller supersonic regions with lower Mach numbers in the divergent section. The pressure decay from the twotube system also occurs more rapidly for this larger throat case.

As a final comparison, the plots in Fig. 8 show results for the large throat case for sequential detonations in both tubes. These results are analogous to the smallthroat calculations in Fig. 6 and the second detonation 
is initiated at approximately the same time as in that case 3. Again the diffracted shock from the lower tube initiates a detonation at the open end in the upper tube. The strength of this induced detonation is nearly the same in both cases. The pressure in the convergent section of the nozzle is, however, considerably lower for the larger throat case. In both cases the pressure and Mach number contours in the upper tube indicate the presence of a series of shocks traveling in transverse directions as the result of interactions between the detonation initiated at the close end and the reaction zone created by the shock entering from the open end.

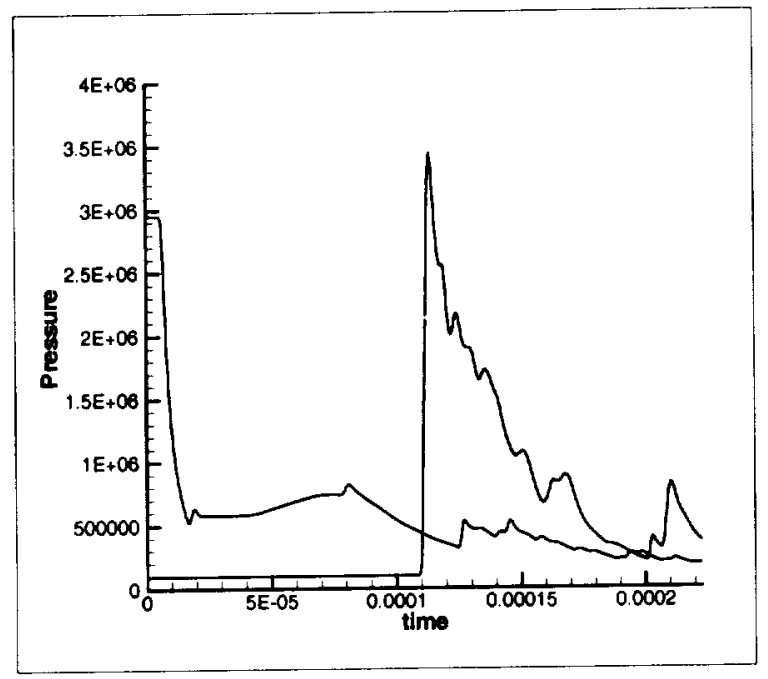

Fig. 9. Time history of pressure on centerline at head end for single pulse case. Large throat area geometry. Curve on left represents lower tube (with detonation); curve on right corresponds to upper tube (no detonation).

Time History in Multi-Tube Calculations: To provide more quantitative interpretations of the above computations we next present some time histories of the pressure at the mid-point of the head end. Figure 9 shows the time history of the pressure for the larger throat configuration with a detonation in only the lower tube. The results show the pressure on the centerline of both tube. The line trace that starts with a large spike on the left is the lower tube in which the detonation is initiated. The initial $30 \mathrm{~atm}$ pressure corresponds to the pressure used for the numerical initiation. The line plot on the right shows the pressure in the upper tube. As can be seen, the pressure in this tube remains at one atmosphere for the first $0.1 \mathrm{~ms}$ during which time the detonation propagates through the lower tube, generates an external shock that expands around the center land, and then propagates upstream into the upper tube. The peak on the right indicates the pressure when this shock reflects off the head wall. Note that this pressure is nearly as high as that generated by the initial detonation. The rate of decay is, however, quite rapid. The introduction of fresh propellants in this tube should most likely be delayed until the pressure level has decreased to near its original level. Again, we note that the size of the throat area in the common nozzle will dictate the rate of decay is both tubes, as well as the ultimate pressure eventually established.

Figure 10 shows similar results for the small throat case. As can be seen from the figures, there is little difference between the two plots, but the decay rates are considerably slower when the throat is smaller. This strongly impacts the maximum pulse repetition rate as well as the average thrust level in the engine.

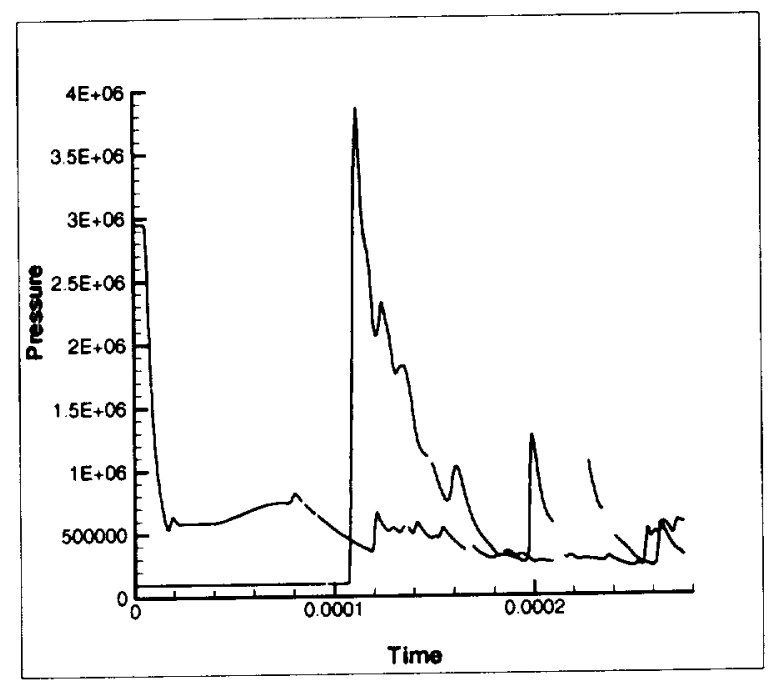

Fig. 10. Time history of pressure on centerline at head end for single pulse case. Small throat area geometry. Curve on left represents lower tube (with detonation); curve on right corresponds to upper tube (no detonation).

\section{Conclusions}

The characteristics of multi-tube PDE's have been investigated by means of a two-dimensional model. The specific multi-tube simulated is composed of two detonation tubes exhausting into a common nozzle. Computations have been performed for two different values of the common nozzle throat area. Multidimensional simulations of single-tube PDE's of different lengths have been performed to obtain a better understanding of the flow field involved. The results show that the pressure induced in adjacent tubes by the detonation from a neighboring tube is nearly as large as that produced by the detonation itself. The rate at which this pressure peak decays depends upon the 
nozzle throat area and the detonation tube length (and volume). The results indicate that the shock wave produced by the detonation is quite capable of initiating high-speed combustion in adjacent tubes if they have all ready been filled with fresh propellants. The likelihood of this increases as the number of tubes in a single engine is increased. Careful analysis of the unsteady flowfields is needed to ensure proper operation of multi-tube PDE systems exhausting through a common nozzle. As contrasted with the inlet where increasing the number of tubes appears to always provide improved operation, the flow in the nozzle section may limit the maximum number of tubes in an engine.

\section{Acknowledgement}

This work was supported by NASA Marshall Space Flight Center under contract number NAS8-97301.

\section{References}

1. Eidelman, S., Grossmann, W., and Lottati, I., "Review of Propulsion Applications and Numerical Simulations of the Pulse Detonation Engine Concept," Journal of Propulsion and Power, Vol. 7, No. 6, pp.857-865, 1991.

2. Bussing, T. R. A. and Pappas, G., "Pulse Detonation Engine Theory and Concepts," Progress in Astronautics and Aeronautics, Eds. Murthy, S. N. B., and Curran, E. T., Vol. 165, pp.421-472, 1996.

3. Kailasanath, K., Patnaik, G., and Li, C "Computational Studies of Pulse Detonation Engines: A Status Report," AIAA Paper 99-2634, June 1999.

4. Cambier, J.-L. and Tegner, J. K., "Strategies for Pulsed Detonation Engine Performance Optimization," Journal of Propulsion and Power, Vol. 14, No. 4, pp.489-498, 1998.
5. Kailasanath, K., "Review of Propulsion Applications of Detonation Waves," AIAA Journal, Vol. 38, No. 9, pp.1698-1708, 2000.

6. Brophy, C. M. and Netzer, "Effects of Ignition Characteristics and Geometry on the Performance of a JP- $10 / \mathrm{O}_{2}$ Fueled Pulse Detonation Engine," AIAA Paper 99-2635, June 1999.

7. Ebrahimi, H. B., and Merkle, C. L., "A Numerical Simulation of the Pulse Detonation Engine with Hydrogen Fuels," AIAA Paper 99-2259, June 1999.

8. Mohanraj, R. and Merkle, C. L., "A Numerical Study of Pulse Detonation Engine Performance," AIAA paper 2000-0315, January 2000.

9. Mohanraj, R., Ebrahimi, H. B., and Merkle, C. L., "Pulse Detonation Rocket Engines," Fourth International symposium on Liquid Space Propulsion, Heilbronn, Germany, Mar. 2000.

10. Ebrahimi, H. B., Mohanraj, R. and Merkle, C. L., "Multi-Level Analysis of Pulsed Detonation Engines," AIAA Paper 2000-3589, July 2000.

11. Talley, D., "The Ideal Constant Volume Limit of Pulsed Propulsion," AIAA paper 2000-3216, July 2000.

12. Li, C., Kailasanath, K., and Patnaik, G., "A Numerical Study of Flow Field Evolution in a Pulsed Detonation Engine," AIAA paper 20000314, January 2000.

13. Bratkovich, T. E., and Bussing, T. R. A., "A Pulse Detonation Engine Performance Model," AIAA paper 95-3155, July 1995.

14. Mohanraj, R, Merkle, C. L., and Houshang B. Ebrahimi "Modeling of Pulse Detonation Engine Operation "AIAA Paper 2001-0475, January 2001. 